\title{
Free Vibration of Pre-Tensioned Electromagnetic Nanobeams
}

\author{
M. Zakaria\& Amira M. Al Harthy \\ Department of Mathematics, Faculty of Science, Al-Baha University, Al-Aqiq, P.O. Box 1988, Kingdom of Saudi \\ Arabia.
}

\begin{abstract}
The transverse free vibration of electromagnetic nanobeams subjected to an initial axial tension based on nonlocal stress theory is presented. It considers the effects of nonlocal stress field on the natural frequencies and vibration modes. The effects of a small-scale parameter at molecular level unavailable in classical macro-beams are investigated for three different types of boundary conditions: simple supports, clamped supports and elastically constrained supports. Analytical solutions for transverse deformation and vibration modes are derived. Through numerical examples, effects of the dimensionless Hartmann number, nano-scale parameter andpre-tension on natural frequencies are presented and discussed.
\end{abstract}

Keywords: Nanobeam, Natural frequency, Nonlocal stress, Pre-tensioned, Vibration, mode, Hartmann number

\section{Introduction}

Recently, research on dynamic behavior ofnano-structures has become a hot field because of the application prospects of nano-electromechanicalsystems (NEMSs) or nano-machine components. Although nano-structures, such as nanobeams and nano belts, have been proposed to have practical applications, analysis in this field has been lacking in particular the dynamics of pre-tensioned nano structures. The relevant researches on transverse vibrations of axially moving macro-beams can be dated back to Mote (1965) constructed mathematical model of axially moving beams firstly based on the Hamilton principle and also determined the first three natural frequencies and modes. His results were confirmed by experiment (Mote and Naguleswaran, 1966). There are several excellent and comprehensive survey papers, notably Simpson (1973) researched the natural frequency and mode functionof axially moving beams without pre-tension and clamped at both ends. Oz et al. (2001) introduced axially moving beams with time-dependent velocity through multiple scale analysis. Liu and Zhang (2007)presented the nonlinear vibration of viscoelastic belts. The bifurcation of transverse vibration for axiallyaccelerating moving strings was investigated by Chenand $\mathrm{Wu}$ (2005).Yang and Chen (2005) addressed dynamic stability problem of axially moving viscoelastic beams.

The nonlocal beam models received increasing interest in the past few years. Nonlocal continuum theories regardthe stress state at a point as a function of the strain states of all points in the body, in contrast to the classical viewthat the stress state at a given point is uniquely determined by the strain state at that same point. Eringen (1972) has proposed a nonlocal continuum mechanics theory to deal with the small-scale structure problems while in classical(local) elasticity, the material particles are assumed to be continuously distributed and the stress tensor at a reference point is uniquely determined by the strain tensor through the algebraic relation of Hooke's law, and, the material particles in nonlocal elasticity are allowed to interact with low-range forces and the algebraic constitutive equation is replaced by a generalized constitutive equation of integral and differential operator type. Previous work shows that the dispersion curves obtained by the nonlocal model are in excellent agreement with those obtained by the Born-Karman theory of lattice dynamics. The dislocation core and cohesive stress predicted by the nonlocal theory are also close to corresponding estimates known in the physics of solids (Eringen 1972, 1983; Eringen and Edelen 1972). The nonlocal elasticity theory was applied in nano mechanics including lattice dispersion of elasticwaves, wave propagation in composites, dislocation mechanics, static deflection, fracture mechanics, and surface tension fluids. (Reddy and Wang, 1998;Peddieson et al., 2003; Zhang et al., 2004; 2005;Wang, 2005; Lu et al., 2006; Wang and Varadan, 2006; Wang et al., 2006; 2008; Xu, 2006; Lim and Wang,2007; Benzair et al., 2008; Kumar et al., 2008; Wangand Duan, 2008). The recent work by Tounsi et al.(2008) concluded that the scale coefficient was radius dependent.

Vibration behavior of beams has been developed for a long time. However, very few papers considernanobeams with nonlocal effects. The nano mechanical vibration of a electromagnetic nanobeam is very different from the classical continuum mechanics theory which deals with the macroscopic scale of a beam. In this paper, we attempt to consider the nonlocal effects of a pre-tensioned electromagnetic nanobeam without axial motion and subsequently study the transverse vibration and the effect of Hartmann parameter of such ananobeam. The model is described by partial differential equations in dimensionless quantities such thatthe analysis is more general and distinctive to describe the difference between electromagnetic nano mechanics and classical mechanics. It is found that pre-tension and nonlocal stress play significant roles in the vibration 
behavior of a electromagnetic nanobeam. Their effects are analyzed and discussed in detail in a few numerical examples.

\section{Problem definition and modeling}

Consider a pre-tensioned nanobeam with the length $L$, initial axial tension $P$ at the ends. The end boundary conditions are arbitrary and will be specified in various cases ofstudy. The force equilibrium for an element of the nano beam. For vibration of a nanobeam, the bending rotation angle with respect to $x$-axis is denoted as $\theta$. Because only small deformation is considered for linear vibration, we have

$\cos \theta=1, \quad \sin \theta=\frac{\partial w}{\partial x}$,

where $w$ is the transverse deformation.

We consider also a constant magnetic field of strength $H_{o}$ acts in the direction of the y. Using Ohm's:

$J=\sigma_{o}\left[E+\mu_{o} U \times H_{o}\right]$.

where $\sigma_{o}$ is the electrical conductivity, $E$ is the intensity vector of the electric field, $\mu_{o}$ is the magnetic permeability, and $U$ is the displacement vector, neglecting the effect of the intensity vector $E$.

The ponder motive force has non-vanishing component directions, given by

$F_{z}=\left(J \times \mu_{o} H_{o}\right)_{z}=\sigma_{o} \mu_{o}^{2} H_{o}^{2} w$,

The equilibrium equation of an element with respect to the $z$-axis can be obtained based on the D'Alembert Principle (Fung,1965) as

$\frac{\partial Q}{\partial x} d x-\left(N \frac{\partial^{2} w}{\partial x^{2}}+\frac{\partial N}{\partial x} \frac{\partial w}{\partial x}\right) d x+\sigma_{o} \mu_{o}^{2} H_{o}^{2} w d x=\rho \frac{\partial^{2} w}{\partial t^{2}}$

where $Q$ is the shear force, $x$ is the axial, $N$ is the internal axial force, $\rho$ is the line density and $t$ is time.

$\frac{\partial M}{\partial x}-Q=0$,

where $M$ isthe bending moment.

ughout, we have (Yang and Lim, 2008)

$\frac{\partial M}{\partial x}-\left(N \frac{\partial^{2} w}{\partial x^{2}}+\frac{\partial N}{\partial x} \frac{\partial w}{\partial x}\right)+\sigma_{o} \mu_{o}^{2} H_{o}^{2} w-\rho \frac{\partial^{2} w}{\partial t^{2}}=0$

which can also be expressed as

$\frac{\partial M}{\partial x}-\frac{\partial}{\partial x}\left(N \frac{\partial w}{\partial x}\right)+\sigma_{o} \mu_{o}^{2} H_{o}^{2} w-\rho \frac{\partial^{2} w}{\partial t^{2}}=0$

For such a nanobeam with a constant, external axial tension $P$ at the ends, we have

$\frac{\partial^{2} M}{\partial x^{2}}-P \frac{\partial^{2} w}{\partial x^{2}}-\rho \frac{\partial^{2} w}{\partial t^{2}}+\sigma_{o} \mu_{o}^{2} H_{o}^{2} w=0$

According to Eringen (1983), the nonlocal stress in a 2D domain can be approximately governed by asecondorder differential equation

$\left[1-\left(e_{o} a\right)^{2} \nabla^{2}\right] \sigma_{i j}=\sigma_{i j}^{\prime}$

where $\sigma_{i j}(i, j=1,2)$ are the nonlocal stresses, $\sigma_{i j}^{\prime}(i, j=1,2)$ the classical local stresses, $e_{o}$ a constant dependenton material, and $a$ an internal characteristic length, e.g., for lattice parameter, C-C bond length. For ananobeam, the governing equation above with respect to the neutral axis can be reduced to an ordinary differential equation as

$\left[1-\left(e_{o} a\right)^{2} \frac{d^{2}}{d x^{2}}\right] \sigma=\sigma^{\prime}$

where $\sigma$ indicates the nonlocal normal stresses while $\sigma$ 'the classical local normal stresses along the $x$-axis.From Eq. (8), the nonlocal normal stresses can be solved and expressed in an infinite series as (Lim andWang, 2007)

$\sigma=-E z \frac{\partial^{2} w}{\partial x^{2}}-E z\left(e_{o} a\right)^{2} \frac{\partial^{4} w}{\partial x^{4}}+\ldots$,

where $E$ is the Young's modulus and $z$ is the transverse coordinate. Integrating Eq. (10) above with respect to thedistance from the neutral axis and over the cross-sectional area, the nonlocal bending moment isgoverned by

$M-\left(e_{o} a\right)^{2} \frac{\partial^{2} M}{\partial x^{2}}=-E I \frac{\partial^{2} w}{\partial x^{2}}$

where $E I$ is the flexural stiffness. From Eqs. (8) and(12), the following governing differential equation ofmotion for a nanobeam subjected to an initial axial tension $P$ can be derived as

$$
\begin{aligned}
\rho \frac{\partial^{2} w}{\partial t^{2}} & +P \frac{\partial^{2} w}{\partial x^{2}}-\sigma_{o} \mu_{o}^{2} H_{o}^{2} w \\
& -\left(e_{o} a\right)^{2}\left(\rho \frac{\partial^{4} w}{\partial x^{2} \partial t^{2}}+P \frac{\partial^{4} w}{\partial x^{4}}-\sigma_{o} \mu_{o}^{2} H_{o}^{2} \frac{\partial^{2} w}{\partial x^{2}}\right)=-E I \frac{\partial^{4} w}{\partial x^{4}}
\end{aligned}
$$

For generality, dimensionless formulation is adopted using the following parameters 
$x^{*}=\frac{x}{L}, \quad w^{*}=\frac{w}{L}, \quad t^{*}=t \sqrt{E I /\left(\rho L^{4}\right)}, \quad H_{a}^{2}=\frac{\sigma_{o} \mu_{o}^{2} H_{o}^{2}}{\rho L}, \quad M^{*}=\frac{M L}{E I}$

where $H_{a}$ is the Hartmann number. In dimensionless quantities, Eq. (13) then becomes, dropping the asterisks for convenience

$\frac{\partial^{2} w}{\partial t^{2}}+\left(\bar{P}+\tau^{2} \bar{H}_{a}^{2}\right) \frac{\partial^{2} w}{\partial x^{2}}-\tau^{2} \frac{\partial^{4} w}{\partial x^{2} \partial t^{2}}+\left(1-\tau^{2} \bar{P}\right) \frac{\partial^{4} w}{\partial x^{4}}-\bar{H}_{a}^{2} w=0$,

where $\tau=e_{o} a / L, \bar{P}=P L^{2} / E I$ and $\bar{H}_{a}^{2}=\rho L^{5} H_{a}^{2} / E I$. From Eqs. (8) and (12), the nonlocal bending momentfor the non-dimensional form can be expressed as

$M=\tau^{2}\left(\frac{\partial^{2} w}{\partial t^{2}}-\bar{P} \frac{\partial^{2} w}{\partial x^{2}}-\bar{H}_{a}^{2} w\right)+\frac{\partial^{2} w}{\partial x^{2}}$,

For linear free vibration of a nanobeam, the modes are harmonic in time. Hence the timedependenttransverse deformation of the nanobeam can be represented by

$$
\begin{aligned}
& w(x, t)=\bar{w}_{n}(x) e^{i \omega_{n} t} \\
& M(x, t)=\bar{M}_{n}(x) e^{i \omega_{n} t}
\end{aligned}
$$

where $\bar{w}_{n}(x)$ is the dimensionless amplitude of vibrationand $n=1,2, \ldots$ denotes the vibration modenumber. Substituting Eq. (16) into Eqs. (14), and (15) the governing equation is transformed into the frequencydomain as $\left(1+\tau^{2}\left(\omega^{2}-\bar{P}\right)\right) \frac{d^{4} \bar{w}_{n}}{d x^{4}}+\left(\bar{P}+\tau^{2} \bar{H}_{a}^{2}\right) \frac{d^{2} \bar{w}_{n}}{d x^{2}}-\left(\bar{H}_{a}^{2}+\omega^{2}\right) \bar{w}_{n}=0$,

$\bar{M}=\left(1-\tau^{2} \bar{P}\right) \frac{d^{2} \bar{w}_{n}}{d x^{2}}-\left(\tau^{2} \omega^{2}+\bar{H}_{a}^{2}\right) \bar{w}_{n}$

Eq. (17) can be factorized as

$\prod_{j=1}^{4}\left(\frac{d}{d x}-k_{j n}\right) \bar{w}_{n}(x)=0$,

where $k_{1 n}, k_{2 n}, k_{3 n}$, and $k_{4 n}$ are the roots of the characteristic equation

$K_{j n}^{4}-A k_{j n}^{2}-B=0$,

where $A=\left(\bar{P}+\tau^{2} \bar{H}_{a}^{2}\right) /\left(1+\tau^{2}\left(\bar{P}-\omega_{n}^{2}\right)\right)$, and $B=\left(\bar{H}_{a}^{2}+\omega_{n}^{2}\right) /\left(1+\tau^{2}\left(\omega_{n}^{2}-\bar{P}\right)\right)$.

Since Eq. (20) is a fourth-order polynomial in terms of $k_{i}$ the four roots are denoted by $k_{n j}(j=1,2,3,4)$, respectively. Because only linear free vibration isconcerned, the superposition of the four solutionswith respect to each root $k_{j}$ is also a solution of Eq. (19). Hence

$\bar{w}_{n}(x)=C_{1 n} e^{i k_{1 n} x}+C_{2 n} e^{i k_{2 n} x}+C_{3 n} e^{i k_{3 n} x}+C_{4 n} e^{i k_{4 n} x}$,

$\bar{M}_{n}(x)=\ell_{1 n} C_{1 n} e^{k_{1 n} x}+\ell_{2 n} C_{2 n} e^{k_{2 n} x}+\ell_{3 n} C_{3 n} e^{k_{3 n} x}+\ell_{4 n} C_{4 n} e^{k_{2 n} x}$,

where $\ell_{j n}=k_{j n}^{2}\left(1-\tau^{2} \bar{P}\right)-\left(\tau^{2} \omega^{2}+\bar{H}_{a}^{2}\right), C_{j n}(j=1,2,3,4)$ are four arbitrary constants ofintegration

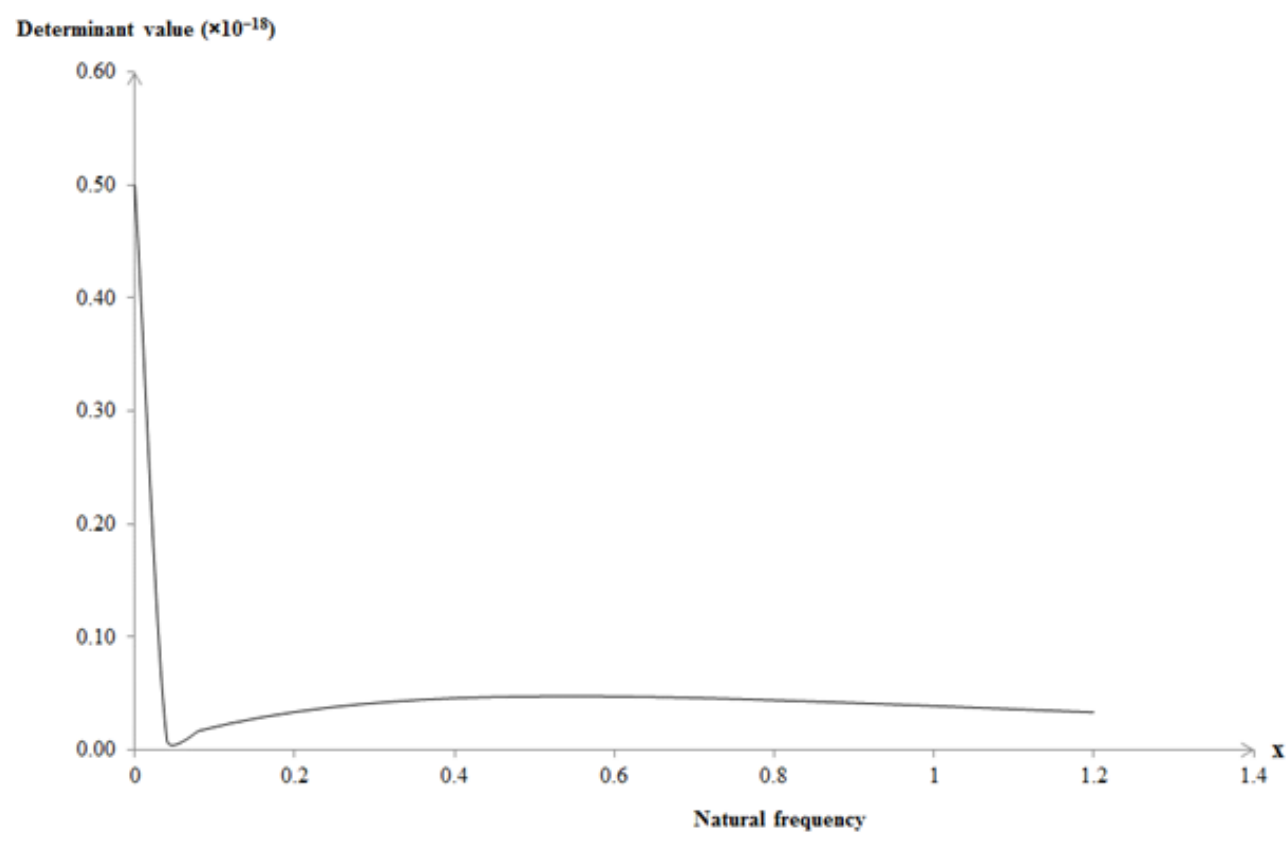

Fig. 1: The relationship between determinant value andnatural frequency. 
associated with Eq. (20) which is afourth-order ordinary differential equation.

\section{Applications and discussion}

To illustrate the effects of nonlocal stress and initial axial tension on the free vibration frequency of a nanobeam, the following examples for various boundary conditions are presented and discussed.

\subsection{Simply supported nanobeams}

For a nanobeam simply supported at both ends, the boundary conditions for the bending moments and displacements are

$M(0, t)=0, \quad M(1, t)=0, \quad w(0, t)=0, \quad w(1, t)=0$.

Substitute Eq. (16) into Eq. (23) and simplify the results. Further substituting Eqs. (21) and (22) into the results obtained above yields

$$
\begin{aligned}
& \left\{\begin{array}{l}
C_{1 n}\left(\ell_{1 n}+\ell_{2 n} \bar{C}_{2 n}+\ell_{3 n} \bar{C}_{3 n}+\ell_{4 n} \bar{C}_{4 n}\right)=0, \\
C_{1 n}\left(\ell_{1 n} e^{i k_{1 n}}+\ell_{2 n} \bar{C}_{2 n} e^{i k_{2 n}}+\ell_{3 n} \bar{C}_{3 n} e^{i k_{3 n}}+\ell_{4 n} \bar{C}_{4 n} e^{i k_{4 n}}\right)=0, \\
C_{1 n}\left(1+\bar{C}_{2 n}+\bar{C}_{3 n}+\bar{C}_{4 n}\right)=0, \\
C_{1 n}\left(e^{i k_{1 n} x}+\bar{C}_{2 n} e^{i k_{2 n}}+\bar{C}_{3 n} e^{i k_{3 n}}+\bar{C}_{4 n} e^{i k_{4 n}}\right)=0,
\end{array}\right. \\
& \text { where } \bar{C}_{j n}=C_{j n} / C_{1 n},(j=1,2,3) \\
& \text { For an arbitrary } C_{1 n} \neq 0 \text { the coefficients inEq. (24) can be obtained as }
\end{aligned}
$$

$$
\begin{aligned}
\bar{C}_{2 n}=\frac{\left(e^{i k_{1 n}}-e^{i k_{2 n}}\right)\left(\ell_{1 n}^{2}-\ell_{3 n}^{2}\right)}{\left(e^{i k_{4 n}}+e^{i k_{2 n}}\right)\left(\ell_{4 n}^{2}+\ell_{3 n}^{2}\right)}+\frac{\left(e^{i k_{1 n}}-e^{i k_{2 n}}\right)\left(\ell_{1 n}^{2}-\ell_{4 n}^{2}\right)}{\left(e^{i k_{2 n}}+e^{i k_{3 n}}\right)\left(\ell_{4 n}^{2}+\ell_{3 n}^{2}\right)}-1 \\
\bar{C}_{3 n}=\frac{\left(e^{i k_{1 n}}-e^{i k_{2 n}}\right)\left(\ell_{1 n}^{2}-\ell_{4 n}^{2}\right)}{\left(e^{i k_{3 n}}+e^{i k_{2 n}}\right)\left(\ell_{4 n}^{2}+\ell_{3 n}^{2}\right)} \\
\bar{C}_{4 n}=\frac{\left(e^{i k_{1 n}}-e^{i k_{2 n}}\right)\left(\ell_{1 n}^{2}-\ell_{3 n}^{2}\right)}{\left(e^{i k_{2 n}}+e^{i k_{4 n}}\right)\left(\ell_{4 n}^{2}+\ell_{3 n}^{2}\right)}
\end{aligned}
$$

Therefore, the $n$-mode amplitude of vibration from Eqs. (21) and (25) is

$$
\begin{aligned}
\bar{w}_{n}(x) & =C_{1 n}\left\{e^{i k_{1 n} x}+\left[\frac{\left(e^{i k_{1 n}}-e^{i k_{2 n}}\right)\left(\ell_{1 n}^{2}-\ell_{3 n}^{2}\right)}{\left(e^{i k_{4 n}}+e^{i k_{2 n}}\right)\left(\ell_{4 n}^{2}+\ell_{3 n}^{2}\right)}\right.\right. \\
& \left.+\frac{\left(e^{i k_{1 n}}-e^{i k_{2 n}}\right)\left(\ell_{1 n}^{2}-\ell_{4 n}^{2}\right)}{\left(e^{i k_{2 n}}+e^{i k_{3 n}}\right)\left(\ell_{4 n}^{2}+\ell_{3 n}^{2}\right)}-1\right] e^{i k_{2 n} x} \\
& \left.+\left[\frac{\left(e^{i k_{1 n}}-e^{i k_{2 n}}\right)\left(\ell_{1 n}^{2}-\ell_{4 n}^{2}\right)}{\left(e^{i k_{3 n}}+e^{i k_{2 n}}\right)\left(\ell_{4 n}^{2}+\ell_{3 n}^{2}\right)}\right] e^{i k_{3 n} x}+\left[\frac{\left(e^{i k_{1 n}}-e^{i k_{2 n}}\right)\left(\ell_{1 n}^{2}-\ell_{3 n}^{2}\right)}{\left(e^{i k_{2 n}}+e^{i k_{4 n}}\right)\left(\ell_{4 n}^{2}+\ell_{3 n}^{2}\right)}\right] e^{i k_{4 n} x}\right\}
\end{aligned}
$$

and the corresponding time-dependent displacement from Eq. (16) is

$$
\begin{aligned}
w_{n}(x, t) & =C_{1 n}\left\{e^{i k_{1 n} x}+\left[\frac{\left(e^{i k_{1 n}}-e^{i k_{2 n}}\right)\left(\ell_{1 n}^{2}-\ell_{3 n}^{2}\right)}{\left(e^{i k_{4 n}}+e^{i k_{2 n}}\right)\left(\ell_{4 n}^{2}+\ell_{3 n}^{2}\right)}\right.\right. \\
& \left.+\frac{\left(e^{i k_{1 n}}-e^{i k_{2 n}}\right)\left(\ell_{1 n}^{2}-\ell_{4 n}^{2}\right)}{\left(e^{i k_{2 n}}+e^{i k_{3 n}}\right)\left(\ell_{4 n}^{2}+\ell_{3 n}^{2}\right)}-1\right] e^{i k_{2 n} x} \\
& +\left[\frac{\left(e^{i k_{1 n}}-e^{i k_{2 n}}\right)\left(\ell_{1 n}^{2}-\ell_{4 n}^{2}\right)}{\left(e^{i k_{3 n}}+e^{i k_{2 n}}\right)\left(\ell_{4 n}^{2}+\ell_{3 n}^{2}\right)}\right] e^{i k_{3 n} x} \\
& \left.+\left[\frac{\left(e^{i k_{1 n}}-e^{i k_{2 n}}\right)\left(\ell_{1 n}^{2}-\ell_{3 n}^{2}\right)}{\left(e^{i k_{2 n}}+e^{i k_{4 n}}\right)\left(\ell_{4 n}^{2}+\ell_{3 n}^{2}\right)}\right] e^{i k_{4 n} x}\right\} e^{i \omega_{n} t}
\end{aligned}
$$

where $C_{1 n} \neq 0$ is an arbitrary constant. For nontrivialsolution of Eq. (21), the determinant of the coefficientmatrix must be zero

$$
\left(\begin{array}{cccc}
1 & 1 & 1 & 1 \\
\mathrm{e}^{\mathrm{i} \mathrm{k}_{1 \mathrm{n}}} & \mathrm{e}^{\mathrm{i} \mathrm{k}_{2 \mathrm{n}}} & \mathrm{e}^{\mathrm{ik_{3n }}} & \mathrm{e}^{\mathrm{i} \mathrm{k}_{4 \mathrm{n}}} \\
\ell_{1 \mathrm{n}} & \ell_{2 \mathrm{n}} & \ell_{3 \mathrm{n}} & \ell_{4 \mathrm{n}} \\
\ell_{1 \mathrm{n}} \mathrm{e}^{\mathrm{i} \mathrm{k}_{1 \mathrm{n}}} & \ell_{2 \mathrm{n}} \mathrm{e}^{\mathrm{ik_{2n }}} & \ell_{3 \mathrm{n}} \mathrm{e}^{\mathrm{i} \mathrm{k}_{3 \mathrm{n}}} & \ell_{4 \mathrm{n}} \mathrm{e}^{\mathrm{ik_{4n }}}
\end{array}\right)=0,
$$

which yields a characteristic equation as

$\ell_{3 n}^{2} \ell_{4 n}^{2}\left(e^{i k_{4 n}}-e^{i k_{3 n}}\right)\left(e^{i k_{2 n}}-e^{i k_{1 n}}\right)+\ell_{2 n}^{2} \ell_{4 n}^{2}\left(e^{i k_{4 n}}-e^{i k_{2 n}}\right)\left(e^{i k_{1 n}}-e^{i k_{3 n}}\right)$ 
$+\ell_{1 n}^{2} \ell_{4 n}^{2}\left(e^{i k_{4 n}}-e^{i k_{1 n}}\right)\left(e^{i k_{3 n}}-e^{i k_{2 n}}\right)+\ell_{2 n}^{2} \ell_{3 n}^{2}\left(e^{i k_{3 n}}-e^{i k_{2 n}}\right)\left(e^{i k_{4 n}}-e^{i k_{1 n}}\right)$
$+\ell_{1 n}^{2} \ell_{3 n}^{2}\left(e^{i k_{3 n}}-e^{i k_{1 n}}\right)\left(e^{i k_{2 n}}-e^{i k_{4 n}}\right)+\ell_{1 n}^{2} \ell_{2 n}^{2}\left(e^{i k_{2 n}}-e^{i k_{1 n}}\right)\left(e^{i k_{4 n}}-e^{i k_{3 n}}\right)=0$

The $n$-mode vibration mode and transverse deformation can be solved to the extent of an arbitrary constant $C_{1 n} \neq 0$. The analysis above can be described clearly through numerical examples. For instance, taking $\tau=0.3$ and $P=2$, the roots for $\omega_{n}(n=1,2, \ldots)$ satisfyingEqs. (18) and (28) can be obtained as the interceptsof the horizontal axis in Fig. 1 where the determinant Eq. (27) vanishes.

It is obvious that there are infinite modes of frequency, which make the determinant zero. The first intercept with the horizontal axis is the fundamental frequency; the second intercept is the second mode frequency, and so on. Following the numerical procedure above, the relationship between $\omega_{1}, \omega_{2}$ and the dimensionless nano scale parameter $\tau$ can be obtained as shown in Fig. 2.

We can find that the fundamental and the second mode frequencies reduce with the increasing $\tau$. Hence, the natural frequencies reduce when the stronger nonlocal stress effect is present. It is also obvious that the frequencies increase with the dimensionless

pre-tension $P$. Obviously, $\tau$ and $P$ affect very much the natural vibration frequencies.

\section{Natural frequencies}

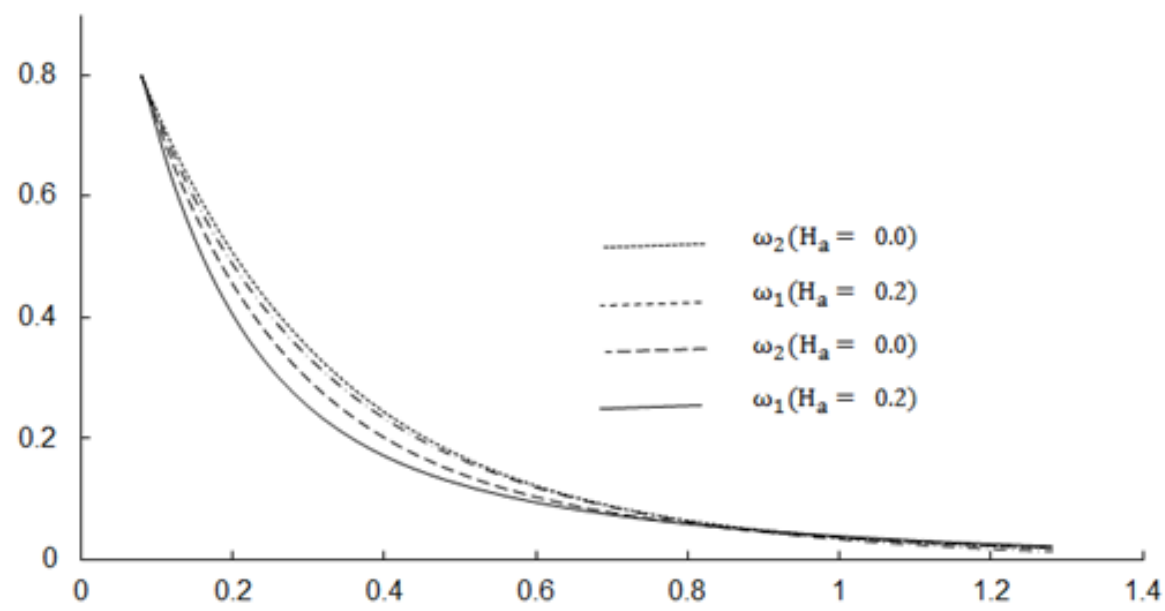

Nonlocal nanoscale parameter

Fig. 2: Nanoscale effects on the first two mode frequencies for clamped nanobeams

\subsection{Clamped nanobeams}

The problem of a clamped, pre-tensioned nano beam is presented in the following example. Theclamped boundary conditions are

$w(o, t)=0, w(1, t)=0, \quad \frac{\partial w(o, t)}{\partial x}=0, \quad \frac{\partial w(1, t)}{\partial x}=0$.

From the above equation, the result can be deduced by Eqs. (16) and (21)

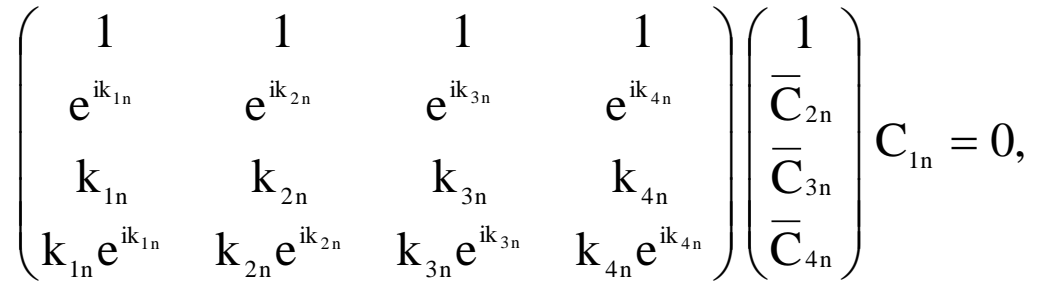

which yields

$$
\begin{aligned}
& \bar{C}_{2 n}=\frac{1}{K}\left\{\begin{array}{c}
\left(e^{i k_{4 n}}-e^{i k_{1 n}}\right)\left(k_{3 n}^{2}-k_{2 n}^{2}\right)+\left(e^{i k_{1 n}}-e^{i k_{3 n}}\right)\left(k_{4 n}^{2}-k_{2 n}^{2}\right) \\
+\left(e^{i k_{4 n}}-e^{i k_{3 n}}\right)\left(k_{2 n}^{2}-k_{1 n}^{2}\right)
\end{array}\right\} \\
& \bar{C}_{3 n}=\frac{\left(e^{i k_{1 n}}-e^{i k_{2 n}}\right)\left(k_{2 n}^{2}-k_{4 n}^{2}\right)+\left(e^{i k_{2 n}}-e^{i k_{4 n}}\right)\left(k_{2 n}^{2}-k_{1 n}^{2}\right)}{\left(e^{i k_{3 n}}-e^{i k_{2 n}}\right)\left(k_{4 n}^{2}-k_{2 n}^{2}\right)+\left(e^{i k_{1 n}}-e^{i k_{4 n}}\right)\left(k_{3 n}^{2}-k_{2 n}^{2}\right)}
\end{aligned}
$$


$\bar{C}_{4 n}=\frac{\left(e^{i k_{1 n}}-e^{i k_{2 n}}\right)\left(k_{3 n}^{2}-k_{2 n}^{2}\right)+\left(e^{i k_{2 n}}-e^{i k_{3 n}}\right)\left(k_{1 n}^{2}-k_{2 n}^{2}\right)}{\left(e^{i k_{3 n}}-e^{i k_{2 n}}\right)\left(k_{4 n}^{2}-k_{2 n}^{2}\right)+\left(e^{i k_{1 n}}-e^{i k_{4 n}}\right)\left(k_{3 n}^{2}-k_{2 n}^{2}\right)}$

where $K=\left(e^{i k_{4 n}}-e^{i k_{1 n}}\right)\left(k_{3 n}^{2}-k_{2 n}^{2}\right)+\left(e^{i k_{1 n}}-e^{i k_{3 n}}\right)\left(k_{4 n}^{2}-k_{2 n}^{2}\right)$

Hence, the $n$-mode amplitude of vibration is

$$
\begin{aligned}
& \bar{w}_{n}(x)=C_{1 n}\left\{e^{i k_{1 n} x}+\left[\frac{\left(e^{i k_{4 n}}-e^{i k_{1 n}}\right)\left(k_{3 n}^{2}-k_{2 n}^{2}\right)}{K}\right.\right. \\
& \left.+\frac{\left(e^{i k_{1 n}}-e^{i k_{3 n}}\right)\left(k_{4 n}^{2}-k_{2 n}^{2}\right)+\left(e^{i k_{4 n}}-e^{i k_{3 n}}\right)\left(k_{2 n}^{2}-k_{1 n}^{2}\right)}{K}\right] e^{k_{2 n} x} \\
& +\left[\frac{\left(e^{i k_{1 n}}-e^{i k_{2 n}}\right)\left(k_{2 n}^{2}-k_{4 n}^{2}\right)+\left(e^{i k_{2 n}}-e^{i k_{4 n}}\right)\left(k_{2 n}^{2}-k_{1 n}^{2}\right)}{\left(e^{i k_{3 n}}-e^{i k_{2 n}}\right)\left(k_{4 n}^{2}-k_{2 n}^{2}\right)+\left(e^{i k_{1 n}}-e^{i k_{4 n}}\right)\left(k_{3 n}^{2}-k_{2 n}^{2}\right)}\right] e^{k_{3 n} x} \\
& \left.+\left[\frac{\left(e^{i k_{1 n}}-e^{i k_{2 n}}\right)\left(k_{3 n}^{2}-k_{2 n}^{2}\right)+\left(e^{i k_{2 n}}-e^{i k_{3 n}}\right)\left(k_{1 n}^{2}-k_{2 n}^{2}\right)}{\left(e^{i k_{3 n}}-e^{i k_{2 n}}\right)\left(k_{4 n}^{2}-k_{2 n}^{2}\right)+\left(e^{i k_{1 n}}-e^{i k_{4 n}}\right)\left(k_{3 n}^{2}-k_{2 n}^{2}\right)}\right] e^{i k_{4 n} x}\right\}
\end{aligned}
$$

and the corresponding time-dependent displacement is

$$
\begin{aligned}
& w_{n}(x, t)=C_{1 n}\left\{e^{i k_{1 n} x}+\left[\frac{\left(e^{i k_{4 n}}-e^{i k_{1 n}}\right)\left(k_{3 n}^{2}-k_{2 n}^{2}\right)}{K}\right.\right. \\
& \left.+\frac{\left(e^{i k_{1 n}}-e^{i k_{3 n}}\right)\left(k_{4 n}^{2}-k_{2 n}^{2}\right)+\left(e^{i k_{4 n}}-e^{i k_{3 n}}\right)\left(k_{2 n}^{2}-k_{1 n}^{2}\right)}{K}\right] e^{i k_{2 n} x} \\
& \quad+\left[\frac{\left(e^{i k_{1 n}}-e^{i k_{2 n}}\right)\left(k_{2 n}^{2}-k_{4 n}^{2}\right)+\left(e^{i k_{2 n}}-e^{i k_{4 n}}\right)\left(k_{2 n}^{2}-k_{1 n}^{2}\right)}{\left(e^{i k_{3 n}}-e^{i k_{2 n}}\right)\left(k_{4 n}^{2}-k_{2 n}^{2}\right)+\left(e^{i k_{1 n}}-e^{i k_{4 n}}\right)\left(k_{3 n}^{2}-k_{2 n}^{2}\right)}\right] e^{i k_{3 n} x} \\
& \left.\quad+\left[\frac{\left(e^{i k_{1 n}}-e^{i k_{2 n}}\right)\left(k_{3 n}^{2}-k_{2 n}^{2}\right)+\left(e^{i k_{2 n}}-e^{i k_{3 n}}\right)\left(k_{1 n}^{2}-k_{2 n}^{2}\right)}{\left(e^{i k_{3 n}}-e^{i k_{2 n}}\right)\left(k_{4 n}^{2}-k_{2 n}^{2}\right)+\left(e^{i k_{1 n}}-e^{i k_{4 n}}\right)\left(k_{3 n}^{2}-k_{2 n}^{2}\right)}\right] e^{i k_{4 n} x}\right\} e^{\omega_{n} t}
\end{aligned}
$$

For nontrivial solution of matrix Eq. (28), the determinant of the coefficient matrix must be zero, or

$k_{3 n} k_{1 n}\left(e^{i k_{4 n}}-e^{i k_{3 n}}\right)\left(e^{i k_{2 n}}-e^{i k_{1 n}}\right)+k_{2 n} k_{4 n}\left(e^{i k_{4 n}}-e^{i k_{2 n}}\right)\left(e^{i k_{1 n}}-e^{i k_{3 n}}\right)$

$$
+k_{1 n} k_{4 n}\left(e^{i k_{3 n}}-e^{i k_{1 n}}\right)\left(e^{i k_{3 n}}-e^{i k_{2 n}}\right)+k_{2 n} k_{3 n}\left(e^{i k_{3 n}}-e^{i k_{2 n}}\right)\left(e^{i k_{4 n}}-e^{i k_{1 n}}\right)
$$

$+k_{1 n} k_{3 n}\left(e^{i k_{3 n}}-e^{i k_{1 n}}\right)\left(e^{i k_{2 n}}-e^{i k_{4 n}}\right)+k_{1 n} k_{2 n}\left(e^{i k_{2 n}}-e^{i k_{1 n}}\right)\left(e^{i k_{4 n}}-e^{i k_{3 n}}\right)=0$.

Analogously, from Eqs. (20) and (34), we cansolve the unknown quantities in Eqs. (32) and (33). Tosolve the problem numerically, the relationship between $\omega_{1}, \omega_{2}$ and $\tau$ is presented in Fig. 3 for two values of $P$. Again, it is obvious that frequency decreases with an increase in $\tau$ while it increases with anincrease in $P$.

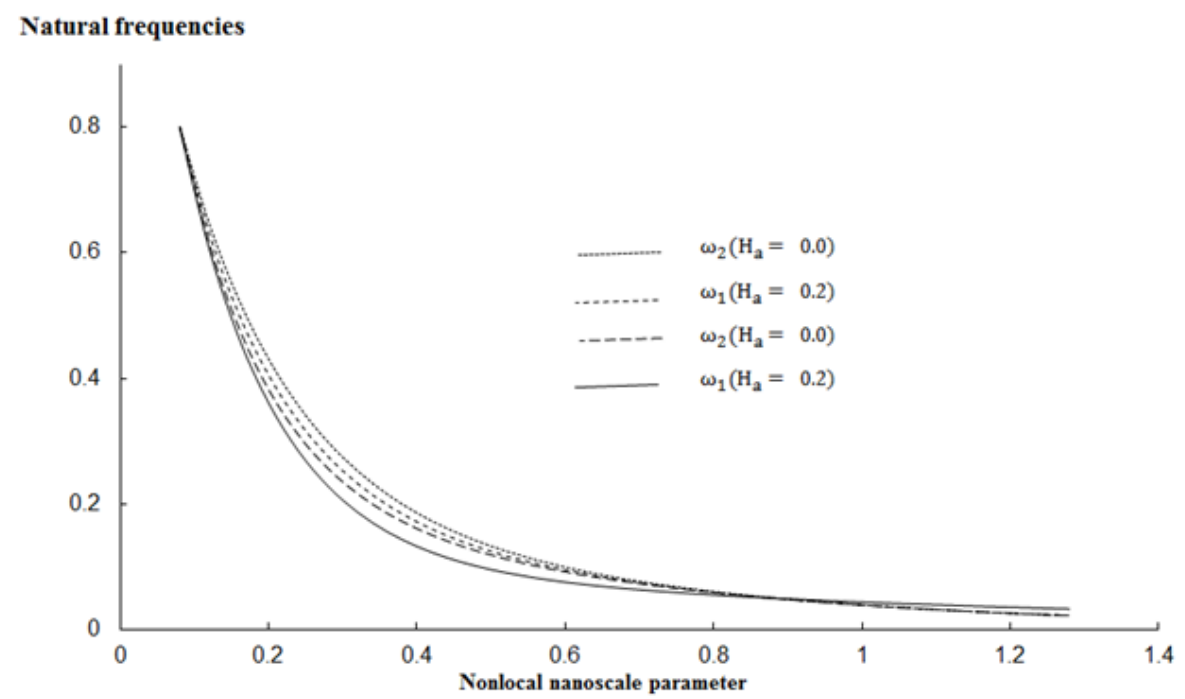

Fig. 3: Nanoscale effects on the first two mode frequencies for clamped nanobeams

\subsection{Nanobeams with elastically constrained ends}

In this example, we consider a special supporting condition for nanobeams with elastically constrained ends (Xie, 2007). The support conditions may be formulated with the following boundary conditions 


$$
\begin{aligned}
& w(0, t)=0, \quad w(1, t)=0, \\
& \frac{\partial^{2} w(0, t)}{\partial x^{2}}-\lambda \frac{\partial w(0, t)}{\partial x}=0, \\
& \frac{\partial^{2} w(1, t)}{\partial x^{2}}-\lambda \frac{\partial w(1, t)}{\partial x}=0,
\end{aligned}
$$

where $\lambda=\bar{\lambda} /(P L)$ is the dimensionless stiffness of theelastically constrained ends in which $\bar{\lambda}$ is the physicalstiffness of the elastic constraint. If $\lambda$ approaches 0 , these ends degenerate to simply supports as discussedin subsection 3.1, while if $\lambda$ approaches infinity, theydegenerate to clamped ones in subsection 3.2.

Substituting Eqs. (16) and (21) into Eq. (35) yields

$A C=0$,

where $\mathrm{A}=$

$$
\begin{aligned}
& \left(\begin{array}{cccc}
1 & 1 & 1 & 1 \\
\mathrm{e}^{\mathrm{k}_{1 \mathrm{n}}} & \mathrm{e}^{\mathrm{k}_{2 n}} & \mathrm{e}^{\mathrm{k}_{3 n}} & \mathrm{e}^{\mathrm{k}_{4 n}} \\
\mathrm{k}_{1 \mathrm{n}}^{2}+\mathrm{i} \lambda \mathrm{k}_{1 \mathrm{n}} & \mathrm{k}_{2 \mathrm{n}}^{2}+\mathrm{i} \lambda \mathrm{k}_{2 \mathrm{n}} & \mathrm{k}_{3 \mathrm{n}}^{2}+\mathrm{i} \lambda \mathrm{k}_{3 \mathrm{n}} & \mathrm{k}_{4 \mathrm{n}}^{2}+\mathrm{i} \lambda \mathrm{k}_{4 \mathrm{n}} \\
\left(\mathrm{k}_{1 \mathrm{n}}^{2}-\mathrm{i} \lambda \mathrm{k}_{1 \mathrm{n}}\right) \mathrm{e}^{\mathrm{ik_{1n }}} & \left(\mathrm{k}_{2 \mathrm{n}}^{2}-\mathrm{i} \lambda \mathrm{k}_{2 \mathrm{n}}\right) \mathrm{e}^{\mathrm{i} \mathrm{k}_{2 \mathrm{n}}} & \left(\mathrm{k}_{3 \mathrm{n}}^{2}-\mathrm{i} \lambda \mathrm{k}_{3 \mathrm{n}}\right) \mathrm{e}^{\mathrm{ik_{3n }}} & \left(\mathrm{k}_{4 \mathrm{n}}^{2}-\mathrm{i} \lambda \mathrm{k}_{4 \mathrm{n}}\right) \mathrm{e}^{\mathrm{i} \mathrm{k}_{4 \mathrm{n}}}
\end{array}\right) \\
& \mathrm{C}=\left(\begin{array}{l}
1 \\
\overline{\mathrm{C}}_{2 \mathrm{n}} \\
\overline{\mathrm{C}}_{3 \mathrm{n}} \\
\overline{\mathrm{C}}_{4 \mathrm{n}}
\end{array}\right) \mathrm{C}_{1 \mathrm{n}}
\end{aligned}
$$

For $C_{1 n} \neq 0$, the following solutions of coefficients are obtained by solving Eq. (37):

$$
\begin{aligned}
& C_{2 n}=\frac{1}{K_{1}}\left\{\begin{array}{c}
\left(e^{i k_{1 n}}-e^{i k_{2 n}}\right)\left(e^{i k_{4 n}}-e^{i k_{3 n}}\right)\left[k_{3 n}-k_{2 n}+i \lambda\left(k_{3 n}-k_{2 n}\right)\right] \\
+\left(e^{i k_{3 n}}-e^{i k_{2 n}}\right)\left(e^{i k_{2 n}}-e^{i k_{4 n}}\right)\left[k_{1 n}-k_{2 n}+i \lambda\left(k_{1 n}-k_{2 n}\right)\right]
\end{array}\right\} \\
& +\frac{e^{i k_{1 n}}-e^{i k_{3 n}}}{e^{i k_{3 n}}-e^{i k_{2 n}}} \\
& C_{3 n}=\frac{1}{K_{1}}\left\{\begin{array}{c}
\left(e^{i k_{4 n}}-e^{i k_{2 n}}\right)\left(e^{i k_{3 n}}-e^{I k_{2 n}}\right)\left[k_{1 n}-k_{2 n}+i \lambda\left(k_{1 n}-k_{2 n}\right)\right] \\
+\left(e^{i k_{4 n}}-e^{i k_{2 n}}\right)\left(e^{i k_{3 n}}-e^{i k_{2 n}}\right)\left[k_{3 n}-k_{2 n}+i \lambda\left(k_{3 n}-k_{2 n}\right)\right]
\end{array}\right\} \\
& +\frac{e^{i k_{2 n}}-e^{i k_{1 n}}}{e^{i k_{3 n}}-e^{i k_{2 n}}} \\
& C_{4 n}=\frac{\left(e^{i k_{1 n}}-e^{i k_{2 n}}\right)\left[k_{3 n}^{2}-k_{2 n}^{2}+i \lambda\left(k_{3 n}-k_{2 n}\right)\right]-\left(e^{i k_{3 n}}-e^{i k_{2 n}}\right)\left[k_{1 n}^{2}-k_{2 n}^{2}+i \lambda\left(k_{1 n}-k_{2 n}\right)\right]}{\left(e^{i k_{3 n}}-e^{i k_{2 n}}\right)\left[k_{4 n}^{2}-k_{2 n}^{2}+i \lambda\left(k_{4 n}-k_{2 n}\right)\right]-\left(e^{i k_{4 n}}-e^{i k_{2 n}}\right)\left[k_{3 n}^{2}-k_{2 n}^{2}+i \lambda\left(k_{3 n}-k_{2 n}\right)\right]^{\prime}} \\
& -\left(e^{i k_{4 n}}-e^{i k_{2 n}}\right)\left(e^{i k_{3 n}}-e^{i k_{2 n}}\right)\left[k_{3 n}^{2}-k_{2 n}^{2}+i \lambda\left(k_{3 n}-k_{2 n}\right)\right] \text {. }
\end{aligned}
$$

where

Then the $n$-mode amplitude of vibration can be obtained as

$$
\begin{aligned}
& \bar{w}_{n}(x)=C_{1 n}\left\{e^{i k_{1 n} x}+\left[\frac{e^{i k_{1 n}}-e^{i k_{3 n}}}{e^{i k_{3 n}}-e^{i k_{2 n}}}\right.\right. \\
& +\frac{1}{K_{1}}\left\{\begin{array}{c}
\left(e^{i k_{1 n}}-e^{i k_{2 n}}\right)\left(e^{i k_{4 n}}-e^{i k_{3 n}}\right)\left[k_{3 n}-k_{2 n}+i \lambda\left(k_{3 n}-k_{2 n}\right)\right] \\
+\left(k_{3 n}-e^{i k_{2 n}}\right)\left(e^{i k_{2 n}}-e^{i k_{4 n}}\right)\left[k_{1 n}-k_{2 n}+i \lambda\left(k_{1 n}-k_{2 n}\right)\right]
\end{array}\right\} e^{k_{2 n} x} \\
& +\left[\frac{e^{i k_{2 n}}-e^{i k_{1} n}}{e^{i k_{3 n}}-e^{i k_{2 n}}}\right. \\
& +\frac{1}{K_{1}}\left\{\begin{array}{c}
\left(e^{i k_{4 n}}-e^{i k_{2 n}}\right)\left(e^{i k_{3 n}}-e^{I k_{2 n}}\right)\left[k_{1 n}-k_{2 n}+i \lambda\left(k_{1 n}-k_{2 n}\right)\right] \\
+\left(e^{i k_{4 n}}-e^{i k_{2 n}}\right)\left(e^{i k_{3 n}}-e^{i k_{2 n}}\right)\left[k_{3 n}-k_{2 n}+i \lambda\left(k_{3 n}-k_{2 n}\right)\right]
\end{array}\right\} e^{k_{3 n} x} \\
& \left.+\left[\frac{\left(e^{i k_{1 n}}-e^{i k_{2 n}}\right)\left[k_{3 n}^{2}-k_{2 n}^{2}+i \lambda\left(k_{3 n}-k_{2 n}\right)\right]-\left(e^{i k_{3 n}}-e^{i k_{2 n}}\right)\left[k_{1 n}^{2}-k_{2 n}^{2}+i \lambda\left(k_{1 n}-k_{2 n}\right)\right]}{\left(e^{i k_{3 n}}-e^{i k_{2 n}}\right)\left[k_{4 n}^{2}-k_{2 n}^{2}+i \lambda\left(k_{4 n}-k_{2 n}\right)\right]-\left(e^{i k_{4 n}}-e^{i k_{2 n}}\right)\left[k_{3 n}^{2}-k_{2 n}^{2}+i \lambda\left(k_{3 n}-k_{2 n}\right)\right]}\right] e^{i k_{4 n} x}\right\}
\end{aligned}
$$

and the corresponding time-dependent displacement is shown in Eq. (40):

$$
\begin{aligned}
w_{n}(x, t)=C_{1 n} & \left\{e^{i k_{1 n} x}+\left[\frac{e^{i k_{1 n}}-e^{i k_{3 n}}}{e^{i k_{3 n}}-e^{i k_{2 n}}}\right.\right. \\
& +\frac{1}{K_{1}}\left\{\begin{array}{c}
\left(e^{i k_{1 n}}-e^{i k_{2 n}}\right)\left(e^{i k_{4 n}}-e^{i k_{3 n}}\right)\left[k_{3 n}-k_{2 n}+i \lambda\left(k_{3 n}-k_{2 n}\right)\right] \\
+\left(e^{i k_{3 n}}-e^{i k_{2 n}}\right)\left(e^{i k_{2 n}}-e^{i k_{4 n}}\right)\left[k_{1 n}-k_{2 n}+i \lambda\left(k_{1 n}-k_{2 n}\right)\right]
\end{array}\right\} e^{k_{2 n} x}
\end{aligned}
$$




$$
\begin{gathered}
+\left[\frac{e^{i k_{2 n}}-e^{i k_{1 n}}}{e^{i k_{3 n}}-e^{i k_{2 n}}}\right. \\
+\frac{1}{K_{1}}\left\{\begin{array}{c}
\left(e^{i k_{4 n}}-e^{i k_{2 n}}\right)\left(e^{i k_{3 n}}-e^{i k_{2 n}}\right)\left[k_{1 n}-k_{2 n}+i \lambda\left(k_{1 n}-k_{2 n}\right)\right] \\
+\left(e^{2 n}-e^{i k_{2 n}}\right)\left(e^{i k_{3 n}}-e^{i k_{2 n}}\right)\left[k_{3 n}-k_{2 n}+i \lambda\left(k_{3 n}-k_{2 n}\right)\right]
\end{array}\right] e^{k_{3 n} x} \\
\left.+\left[\frac{\left(e^{i k_{1 n}}-e^{i k_{2 n}}\right)\left[k_{3 n}^{2}-k_{2 n}^{2}+i \lambda\left(k_{3 n}-k_{2 n}\right)\right]-\left(e^{i k_{3 n}}-e^{i k_{2 n}}\right)\left[k_{1 n}^{2}-k_{2 n}^{2}+i \lambda\left(k_{1 n}-k_{2 n}\right)\right]}{\left(e^{i k_{3 n}}-e^{i k_{2 n}}\right)\left[k_{4 n}^{2}-k_{2 n}^{2}+i \lambda\left(k_{4 n}-k_{2 n}\right)\right]-\left(e^{i k_{4 n}}-e^{i k_{2 n}}\right)\left[k_{3 n}^{2}-k_{2 n}^{2}+i \lambda\left(k_{3 n}-k_{2 n}\right)\right]}\right] e^{i k_{4 n} x}\right\} e^{\omega_{n} t}
\end{gathered}
$$

For nontrivial solution of Eq. (36), the determinant of the coefficient matrix must be zero, or Eq. (40)

$k_{3 n} k_{4 n}\left(e^{i k_{2 n}}-e^{i k_{1 n}}\right)\left[\left(k_{3 n}+i \lambda\right)\left(k_{4 n}-i \lambda\right)-\left(k_{4 n}+i \lambda\right)\left(k_{3 n}-i \lambda\right)\right]$

$k_{2 n} k_{4 n}\left(e^{i k_{4 n}}-e^{i k_{1 n}}\right)\left[\left(k_{2 n}+i \lambda\right)\left(k_{4 n}-i \lambda\right)-\left(k_{4 n}+i \lambda\right)\left(k_{2 n}-i \lambda\right)\right]$

$k_{2 n} k_{3 n}\left(e^{i k_{4 n}}-e^{i k_{1 n}}\right)\left[\left(k_{2 n}+i \lambda\right)\left(k_{3 n}-i \lambda\right)-\left(k_{3 n}+i \lambda\right)\left(k_{2 n}-i \lambda\right)\right]$

$k_{1 n} k_{4 n}\left(e^{i k_{3 n}}-e^{i k_{2 n}}\right)\left[\left(k_{1 n}+i \lambda\right)\left(k_{4 n}-i \lambda\right)-\left(k_{4 n}+i \lambda\right)\left(k_{1 n}-i \lambda\right)\right]$

$k_{1 n} k_{3 n}\left(e^{i k_{2 n}}-e^{i k_{4 n}}\right)\left[\left(k_{1 n}+i \lambda\right)\left(k_{3 n}-i \lambda\right)-\left(k_{3 n}+i \lambda\right)\left(k_{1 n}-i \lambda\right)\right]$

$k_{1 n} k_{2 n}\left(e^{i k_{4 n}}-e^{i k_{3 n}}\right)\left[\left(k_{1 n}+i \lambda\right)\left(k_{2 n}-i \lambda\right)-\left(k_{2 n}+i \lambda\right)\left(k_{1 n}-i \lambda\right)\right]$

The relationship between the natural frequencies $\omega_{1}, \omega_{2}$ and nanoscale parameter $\tau$ is Fig. 4 for $k=0.2$. Again, we observe similar effects of $\tau$ and $P$ where increases in $\tau$ and $P$ cause thefrequencies to decrease and increase, respectively.

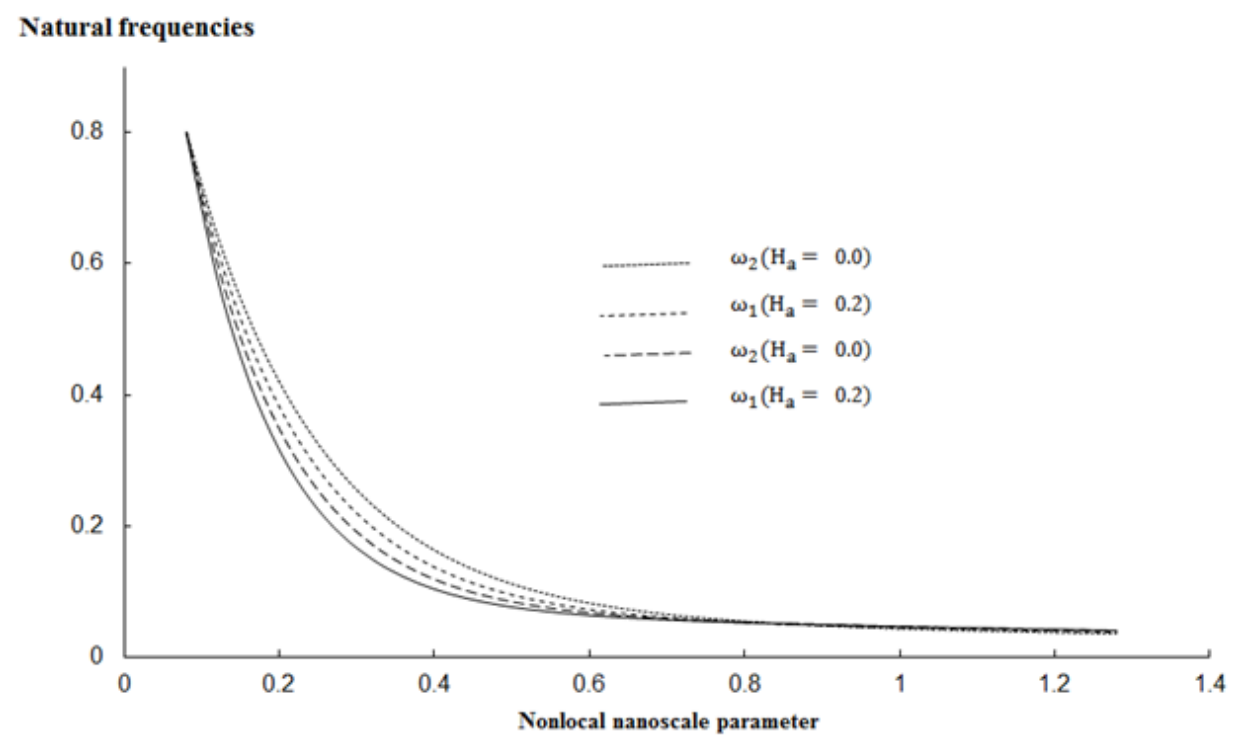

Fig. 4: Nanoscale effects on the first two mode frequencies for nanobeams with elastically constrained ends

\section{Conclusion}

In this paper, we concluded that the transverse free vibration of electromagnetic nanobeam is significantly influenced by the existence of a pre-tension and the dimensionless nano scale parameter. Three numerical examples are presented which include simply supported nano beams, clamped nanobeams and nano beams with elastically constrained ends. In the numerical examples, we find that the first two modefrequencies drop quickly with increasing dimensionless nano scale parameter. On the contrary, thefirst two mode frequencies increase with increasing pre-tension. The effects are similar for the three examples investigated.

\section{References}

[1]. Mote, C.D.Jr., 1965. A study of band saw vibrations. Journal of the Franklin Institute, 279(6):430-444. [doi:10.1016/00160032(65)90273-5]

[2]. Mote, C.D.Jr., Naguleswaran, S., 1966. Theoretical and experimental band saw vibrations. ASME Journal of Engineering Industry, $88(2): 151-156$

[3]. Simpson, A., 1973. Transverse modes and frequencies of beams translating between fixed end supports. Journal of Mechanical Engineering Science, 15(3):159-164.[doi:10.1243/JMES_JOUR_1973_015_031_02]

[4]. Oz, H.R., Pakdemirli, M., Boyaci, H., 2001. Non-linear vibrations and stability of an axially moving beam with time-dependent velocity. International Journal of Non-Linear Mechanics, 36(1):107-115. [doi:10.1016/S0020-7462(99)00090-6]

[5]. Liu, Y.Q., Zhang, W., 2007. Transverse nonlinear dynamical characteristic of viscoelastic belt. Journal of Beijing University of Technology, 33(11):10-13.

[6]. Chen, L.Q., Wu, J., 2005. Bifurcation in transverse vibration ofaxially accelerating viscoelastic strings. Acta Mechanic a Solida Sinica, 26(1):83-86.

[7]. Yang, X.D., Chen, L.Q., 2005. Dynamic stability of axially moving viscoelastic beams with pulsating speed. Applied Mathematics and Mechanics, 26(8):905-910. 
[8]. Eringen AC (1972) Nonlocal polar elastic continua. Int. J Eng. Sci. 10:1-16

[9]. Eringen AC (1983) On differential equations of nonlocal elasticity and solutions of screw dislocation and surface waves. J Appl. Phys. 54:4703-4710

[10]. Eringen AC, Edelen DGB (1972) On nonlocal elasticity. Int. J Eng. Sci. 10:233-248

[11]. Reddy, J.N., Wang, C.M., 1998. Deflection relationships between classical and third-order plate theories. Acta Mechanica Sinica, 130(3):199-208.

[12]. Peddieson, J., Buchanan, G.R., McNitt, R.P., 2003. Application of nonlocal continuum models to nanotechnology. InternationalJournal of Engineering Science, 41(3-5):305-312. [doi:10.1016/S0020-7225(02)00210-0]

[13]. Zhang, Y.Q., Liu, G.R., Wang, J.S., 2004. Small-scale effects on buckling of multiwall carbon nanotubes under axial compression. Physical Review B, 70(20):205430. [doi:10.1103/PhysRevB.70.205430]

[14]. Zhang, Y.Q., Liu, G.R., Xie, X.Y., 2005. Free transverse vibrations of double-walled carbon nanotubes using a theory of nonlocal elasticity. Physical Review B, 71(19):195404. [doi:10.1103/PhysRevB.71.195404]

[15]. Wang, Q., 2005. Wave propagation in carbon nanotubes via nonlocal continuum mechanics. Journal of Applied Physics, 98(12):124301. [doi:10.1063/ 1.2141648]

[16]. Lu, P., Lee, H.P., Lu, C., Zhang, P.Q., 2006. Dynamic properties of flexural beams using a nonlocal elasticity model. Journal of Applied Physics, 99(7):073510. [doi:10.1063/1.2189213]

[17]. Wang, Q., Varadan, V.K., 2006. Vibration of carbon nanotubes studied using nonlocal continuum mechanics. Smart Materials and Structures, 15(2):659-666. [doi:10.1088/0964-1726/15/2/050]

[18]. Wang, C.M., Zhang, Y.Y., Ramesh, S.S., Kitipornchai, S., 2006.Buckling analysis of micro- and nano-rods/tubes based on nonlocal Timoshenko beam theory. Journal of Applied Physics, 39(17):3904-3909. [doi:10.1088/0022-3727/39/17/029]

[19]. Wang, C.M., Kitipornchai, S., Lim, C.W., Eisenberger, M.,2008. Beam bending solutions based on nonlocal Timoshenko beam theory. Journal of Engineering Mechanics, ASCE, 134(6):475-481. [doi:10.1061/(ASCE)0733-9399(2008)134:6(475)]

[20]. Xu, M.T., 2006. Free transverse vibrations of nano-to-micro scale beams. Proceedings of the Royal Society A: Mathematical Physical and Engineering Sciences,462(2074):2977-2995. [doi:10.1098/rspa.2006.1712]

[21]. Lim, C.W., Wang, C.M., 2007. Exact vibrational non local stress modeling with asymptotic higher-order strain gradients for nanobeams. Journal of Applied Physics, 101(5):054312. [doi:10.1063/1.2435878]

[22]. Benzair, A., Tounsi, A., Besseghier, A., Heireche, H., Moulay,N., Boumia, L., 2008.The thermal effect on vibration of singlewalled carbon nanotubes using nonlocal Timoshenko beam theory. Journal of Physics D: Applied Physics, 41(22):225404. [doi:10.1088/0022-3727/41/22/225404]

[23]. Kumar, D., Heinrich, C., Wass, A.M., 2008. Buckling analysis of carbon nanotubes modeled using nonlocal continuum theories. Journal of Applied Physics, 103(7):073521.[doi:10.1063/1.2901201]

[24]. Wang, C.M., Duan, W.H., 2008. Free vibration of Nano rings/arches based on nonlocal elasticity. Journal of Applied Physics, 104(1):014303. [doi:10.1063/1.2951642]

[25]. Fung, Y.C., 1965.Foundations of Solid Mechanics. Prentice-Hall, Englewood Cliffs, NJ, USA, p.305-359.

[26]. Yang, X.D., Lim, C.W., 2008. Nonlinear Vibrations ofNano-beams Accounting for Nonlocal Effect. Fourth Jiangsu-Hong Kong Forum on Mechanics and Its Application, Suzhou, China. Jiangsu Society of Mechanics, Nanjing, p.16-17.

[27]. Xie, G.M., 2007. Vibration Mechanics. National Defense Industry Press, Beijing, China, p.198-203 (in Chinese). 